\title{
STABILITY OF DIFFERENCE ANALOGUE OF LINEAR MATHEMATICAL INVERTED PENDULUM
}

\section{SHAIKHET}

Received 3 May 2005 and in revised form 4 July 2005

A sufficient condition to preserve the property of asymptotic stability for a difference analogue of the linear mathematical inverted pendulum is obtained.

\section{Statement of the problem}

To use numerical investigation of functional differential equations it is very important to know if difference analogues of the considered differential equations have the reliability to preserve some general properties of these equations, in particular, property of stability. This problem is considered here by investigation of a difference analogue of the linear mathematical inverted pendulum.

The problem of stabilization of the mathematical inverted pendulum is very popular among the researches (see, for instance $[1,2,3,5,13,14]$ ). The linearized mathematical model of the controlled inverted pendulum can be described by the following linear differential equation of second order

$$
\ddot{x}(t)-a x(t)=u(t), \quad a>0, t \geq 0 .
$$

The classical way of stabilization of system (1.1) uses the control $u(t)=-b_{1} x(t)-b_{2} \dot{x}(t)$, where $b_{1}>a, b_{2}>0$. But this type of control which represents an instantaneous feedback is quite difficult to realize because usually we need some finite time to make measurements of the coordinates and velocities, to treat the results of the measurements and to implement them in the control action.

Unlike of the classical way of stabilization in which the stabilized control is a linear combination of the state and velocity of the pendulum another way of stabilization was proposed in [4]. There it was supposed that only the trajectory of the pendulum can be observed and stabilized control depends on the whole trajectory of the pendulum, that is

$$
u(t)=\int_{0}^{\infty} d K(\tau) x(t-\tau)
$$


where the kernel $K(\tau)$ is a function of bounded variation on $[0, \infty]$ and the integral is understood in the Stiltjes sense. It means, in particular, that both distributed and discrete delays can be used depending on the concrete choice of the kernel $K(\tau)$. The initial condition for the system (1.1), (1.2) has the form

$$
x(s)=\varphi(s), \quad \dot{x}(s)=\dot{\varphi}(s), \quad s \leq 0,
$$

where $\varphi(s)$ is a given continuously differentiable function.

Definition 1.1. The trivial solution of system (1.1)-(1.3) is called stable if for any $\epsilon>0$ there exists $\delta>0$ such that $\max \{|x(t)|,|\dot{x}(t)|\}<\epsilon$ for all $t \geq 0$ if $\|\varphi\|=\sup _{s \leq 0}(|\varphi(s)|+$ $|\dot{\varphi}(s)|)<\delta$. If, besides, $\lim _{t \rightarrow \infty} x(t)=0$ and $\lim _{t \rightarrow \infty} \dot{x}(t)=0$ for every initial function $\varphi$, then the trivial solution of system (1.1)-(1.3) is called asymptotically stable.

Put

$$
a_{1}=-a-k_{0}, \quad k_{i}=\int_{0}^{\infty} \tau^{i} d K(\tau), \quad i=0,1, \quad \hat{k}_{2}=\int_{0}^{\infty} \tau^{2}|d K(\tau)| .
$$

Theorem 1.2 (see [4]). Let

$$
\begin{gathered}
a_{1}>0, \quad k_{1}>0, \\
\hat{k}_{2}<k_{m}=\frac{4}{1+\sqrt{1+\left(\left(1+a_{1}\right) / k_{1}\right)^{2}}} .
\end{gathered}
$$

Then the trivial solution of system (1.1)-(1.3) is asymptotically stable.

It is shown also [4] that inequalities (1.5) are necessary conditions for asymptotic stability of the trivial solution of system (1.1)-(1.3) but inequality (1.6) is only sufficient one.

Below the mathematical model of the controlled inverted pendulum (1.1)-(1.3) is considered in the following simple form

$$
\ddot{x}(t)-a x(t)=b_{1} x\left(t-h_{1}\right)+b_{2} x\left(t-h_{2}\right), \quad t \geq 0 .
$$

Here $a>0, b_{1}, b_{2}, h_{1}>0, h_{2}>0$ are given arbitrary numbers. From (1.4) it follows that for equation (1.7)

$$
k_{0}=b_{1}+b_{2}, \quad k_{1}=b_{1} h_{1}+b_{2} h_{2}, \quad \hat{k}_{2}=\left|b_{1}\right| h_{1}^{2}+\left|b_{2}\right| h_{2}^{2} .
$$

The main conclusion of our investigation here can be formulated in the following way: if conditions (1.5), (1.6) hold then the trivial solution of equation (1.7) is asymptotically stable and there exists enough small step of discretization of this equation that the trivial solution of the corresponding difference equation is asymptotically stable too.

Note, that the conditions for asymptotic stability are obtained here by virtue of Kolmanovskii and Shaikhet's general method of Lyapunov functionals construction $[6,7,8$, $9,10,11,12,15]$ which is applicable for both differential and difference equations, both for deterministic and stochastic systems with delay. 


\section{Construction of difference analogue}

Transform equation (1.7) to a system of the equations

$$
\dot{x}(t)=y(t), \quad \dot{y}(t)=a x(t)+\sum_{l=1}^{2} b_{l} x\left(t-h_{l}\right) .
$$

To construct a difference analogue of system (2.1) put

$$
x_{i}=x\left(t_{i}\right), \quad t_{i}=i \tau, \quad h_{1}=m_{1} \tau, \quad h_{2}=m_{2} \tau, \quad \tau>0 .
$$

A difference analogue of system (2.1) can be considered in the form

$$
x_{i+1}=x_{i}+\tau y_{i}, \quad y_{i+1}=y_{i}+\tau\left(a x_{i}+\sum_{l=1}^{2} b_{l} x_{i-m_{l}}\right) .
$$

From the first equation of system (2.3) we have

$$
x_{i}=x_{i-m_{l}}+\tau \sum_{j=i-m_{l}}^{i-1} y_{i}, \quad l=1,2 .
$$

From here and (1.8) it follows

$$
\sum_{l=1}^{2} b_{l} x_{i-m_{l}}=k_{0} x_{i}-\tau \sum_{l=1}^{2} b_{l} \sum_{j=i-m_{l}}^{i-1} y_{i}
$$

Substituting (2.5) into the second equation of system (2.3) and using (1.4) we obtain

$$
y_{i+1}=y_{i}-\tau a_{1} x_{i}-\tau^{2} \sum_{l=1}^{2} b_{l} \sum_{j=i-m_{l}}^{i-1} y_{j}
$$

Put

$$
F_{i}=\tau^{2} \sum_{l=1}^{2} b_{l} \sum_{j=i-m_{l}}^{i-1}\left(j-i+1+m_{l}\right) y_{j}, \quad q_{1}=b_{1} m_{1}+b_{2} m_{2}=\tau^{-1} k_{1} .
$$

Calculating $\Delta F_{i}=F_{i+1}-F_{i}$, we have

$$
\begin{aligned}
\Delta F_{i} & =\tau^{2} \sum_{l=1}^{2} b_{l}\left[\sum_{j=i+1-m_{l}}^{i}\left(j-i+m_{l}\right) y_{j}-\sum_{j=i-m_{l}}^{i-1}\left(j-i+1+m_{l}\right) y_{j}\right] \\
& =\tau^{2} \sum_{l=1}^{2} b_{l}\left(m_{l} y_{i}-\sum_{j=i-m_{l}}^{i-1} y_{i}\right)=\tau\left(k_{1} y_{i}-\tau \sum_{l=1}^{2} b_{l} \sum_{j=i-m_{l}}^{i-1} y_{j}\right) .
\end{aligned}
$$

From here and (2.6) it follows

$$
y_{i+1}=-\tau a_{1} x_{i}+\left(1-\tau k_{1}\right) y_{i}+\Delta F_{i}
$$


218 Stability of inverted pendulum

So, system (2.3) can be written in the matrix form

$$
z(i+1)=A z(i)+\Delta F(i)
$$

where

$$
z(i)=\left(\begin{array}{c}
x_{i} \\
y_{i}
\end{array}\right), \quad F(i)=\left(\begin{array}{c}
0 \\
F_{i}
\end{array}\right), \quad A=\left(\begin{array}{cc}
1 & \tau \\
-\tau a_{1} & 1-\tau k_{1}
\end{array}\right) .
$$

\section{Stability conditions of the auxiliary equation}

Following the general method of Lyapunov functionals construction [7] at first consider the auxiliary equation

$$
z(i+1)=A z(i)
$$

which can be written in a scalar form

$$
x_{i+2}=A_{0} x_{i+1}+A_{1} x_{i}
$$

with

$$
A_{0}=2-\tau k_{1}, \quad A_{1}=\tau\left(k_{1}-\tau a_{1}\right)-1
$$

It is well known [15] that necessary and sufficient conditions for asymptotic stability of the trivial solution of equation (3.2) have the form

$$
\left|A_{1}\right|<1, \quad\left|A_{0}\right|<1-A_{1} .
$$

For $A_{1}$ from (3.3), (3.4) it follows $0<\tau\left(k_{1}-\tau a_{1}\right)<2$. It means that

$$
\begin{gathered}
\tau \in\left(0, \frac{k_{1}}{a_{1}}\right), \quad \text { if } k_{1}^{2}<8 a_{1}, \\
\tau \in\left(0, \frac{k_{1}-\sqrt{k_{1}^{2}-8 a_{1}}}{2 a_{1}}\right) \cup\left(\frac{k_{1}+\sqrt{k_{1}^{2}-8 a_{1}}}{2 a_{1}}, \frac{k_{1}}{a_{1}}\right), \quad \text { if } k_{1}^{2} \geq 8 a_{1} .
\end{gathered}
$$

For $A_{0}$ from (3.3), (3.4) it follows $a_{1} \tau^{2}-2 k_{1} \tau+4>0$. It means that

$$
\begin{gathered}
\tau \in(0, \infty), \quad \text { if } k_{1}^{2}<4 a_{1}, \\
\tau \in\left(0, \frac{k_{1}-\sqrt{k_{1}^{2}-4 a_{1}}}{a_{1}}\right) \cup\left(\frac{k_{1}+\sqrt{k_{1}^{2}-4 a_{1}}}{a_{1}}, \infty\right), \quad \text { if } k_{1}^{2} \geq 4 a_{1} .
\end{gathered}
$$

As a result we obtain necessary and sufficient conditions for asymptotic stability of the trivial solution of auxiliary equation (3.2) in the form

$$
0<\tau< \begin{cases}a_{1}^{-1} k_{1}, & k_{1}^{2}<4 a_{1}, \\ a_{1}^{-1}\left(k_{1}-\sqrt{k_{1}^{2}-4 a_{1}}\right), & k_{1}^{2} \geq 4 a_{1} .\end{cases}
$$


Note that if for arbitrary positive definite matrix $C$ the matrix equation

$$
A^{\prime} D A-D=-C
$$

has a positive definite solution $D$ then the function $v(i)=z^{\prime}(i) D z(i)$ is a Lyapunov function for equation (3.1), that is $\Delta v(i)=-z^{\prime}(i) C z(i)$.

Let matrix $C$ be a diagonal matrix with positive elements $c_{1}$ and $c_{2}$. Then the elements $d_{i j}$ of the matrix $D$ satisfy the system of the equation

$$
\begin{gathered}
\tau^{2} a_{1}^{2} d_{22}-2 \tau a_{1} d_{12}=-c_{1}, \\
d_{11}-\left(\tau a_{1}+k_{1}\right) d_{12}-a_{1}\left(1-\tau k_{1}\right) d_{22}=0, \\
\tau^{2} d_{11}+2 \tau\left(1-\tau k_{1}\right) d_{12}-\tau k_{1}\left(2-\tau k_{1}\right) d_{22}=-c_{2},
\end{gathered}
$$

with the solution

$$
\begin{gathered}
d_{11}=\frac{c_{1}\left(a_{1} \tau+k_{1}\right)}{2 a_{1} \tau}+\frac{2-\tau\left(k_{1}-a_{1} \tau\right)}{2} a_{1} d_{22}, \\
d_{12}=\frac{c_{1}}{2 \tau a_{1}}+\frac{\tau a_{1}}{2} d_{22}, \quad d_{22}=\frac{c_{1}\left[2-\tau\left(k_{1}-a_{1} \tau\right)\right]+2 a_{1} c_{2}}{\tau a_{1}\left(k_{1}-a_{1} \tau\right)\left[4-\tau\left(2 k_{1}-a_{1} \tau\right)\right]} .
\end{gathered}
$$

Remark 3.1. Note that without loss of generality in (3.10) we can put $c_{1}=1, c_{2}=c$. Really, if it is not so we can divide matrix equation (3.8) on $c_{1}$. As a result we obtain a new diagonal matrix $C$ with the elements 1 and $c=c_{2} / c_{1}$ and a new matrix $D$ with the elements

$$
\begin{gathered}
d_{11}=\frac{a_{1} \tau+k_{1}}{2 a_{1} \tau}+\frac{2-\tau\left(k_{1}-a_{1} \tau\right)}{2} a_{1} d_{22}, \\
d_{12}=\frac{1}{2 \tau a_{1}}+\frac{\tau a_{1}}{2} d_{22}, \quad d_{22}=\frac{2-\tau\left(k_{1}-a_{1} \tau\right)+2 a_{1} c}{\tau a_{1}\left(k_{1}-a_{1} \tau\right)\left[4-\tau\left(2 k_{1}-a_{1} \tau\right)\right]}
\end{gathered}
$$

Remark 3.2. It is easy to check that by condition (3.7) the matrix $D$ with elements (3.11) is a positive definite one.

\section{Stability conditions of the difference analogue}

Let us obtain now a sufficient condition for asymptotic stability of the trivial solution of (2.10). Transform this equation to the form

$$
z(i+1)-F(i+1)=A z(i)-F(i)
$$

Following the general method of Lyapunov functionals construction [7] we will construct Lyapunov functional $V_{i}$ for equation (2.10) in the form $V_{i}=V_{1 i}+V_{2 i}$, where

$$
V_{1 i}=(z(i)-F(i))^{\prime} D(z(i)-F(i))
$$

and the matrix $D$ is a positive definite solution of matrix equation (3.8). 
220 Stability of inverted pendulum

Calculating $\Delta V_{1 i}$ via (4.2), (4.1), (3.8) we have

$$
\begin{aligned}
\Delta V_{1 i}= & (z(i+1)-F(i+1))^{\prime} D(z(i+1)-F(i+1)) \\
& -(z(i)-F(i))^{\prime} D(z(i)-F(i)) \\
= & (A z(i)-F(i))^{\prime} D(A z(i)-F(i))-(z(i)-F(i))^{\prime} D(z(i)-F(i)) \\
= & -z^{\prime}(i) C z(i)-2 F^{\prime}(i) D(A-I) z(i) .
\end{aligned}
$$

Note that

$$
\begin{aligned}
2 F^{\prime}(i) & D(A-I) z(i) \\
= & 2\left(\begin{array}{ll}
0 & F_{i}
\end{array}\right)\left(\begin{array}{ll}
d_{11} & d_{12} \\
d_{12} & d_{22}
\end{array}\right)\left(\begin{array}{cc}
0 & \tau \\
-\tau a_{1} & -\tau k_{1}
\end{array}\right)\left(\begin{array}{l}
x_{i} \\
y_{i}
\end{array}\right) \\
= & 2 F_{i}\left(\begin{array}{ll}
d_{12} & d_{22}
\end{array}\right)\left(\begin{array}{c}
\tau y_{i} \\
-\tau\left(a_{1} x_{i}+k_{1} y_{i}\right)
\end{array}\right) \\
= & 2 F_{i}\left(-\tau a_{1} d_{22} x_{i}+\tau\left(d_{12}-k_{1} d_{22}\right) y_{i}\right) \\
= & -2 \tau a_{1} d_{22} x_{i} F_{i}+2 \tau\left(d_{12}-k_{1} d_{22}\right) y_{i} F_{i} .
\end{aligned}
$$

Put

$$
\alpha=\frac{2-\tau\left(k_{1}-a_{1} \tau\right)+2 a_{1} c}{\left(k_{1}-a_{1} \tau\right)\left[4-\tau\left(2 k_{1}-a_{1} \tau\right)\right]} .
$$

Then from (3.11), (4.5) it follows

$$
d_{22}=\frac{\alpha}{\tau a_{1}} .
$$

Using (3.11), (4.5), (4.6) we obtain

$$
\begin{aligned}
\tau\left(d_{12}-k_{1} d_{22}\right) & =\frac{1}{2 a_{1}}\left(1-\alpha\left(2 k_{1}-a_{1} \tau\right)\right) \\
& =\frac{1}{2 a_{1}}\left(1-\frac{\left(2 k_{1}-a_{1} \tau\right)\left[2-\tau\left(k_{1}-a_{1} \tau\right)+2 a_{1} c\right]}{\left(k_{1}-a_{1} \tau\right)\left[4-\tau\left(2 k_{1}-a_{1} \tau\right)\right]}\right)=-\beta,
\end{aligned}
$$

where

$$
\beta=\frac{\tau+c\left(2 k_{1}-a_{1} \tau\right)}{\left(k_{1}-a_{1} \tau\right)\left[4-\tau\left(2 k_{1}-a_{1} \tau\right)\right]} .
$$

So, via Remark 3.1, (4.3), (4.4), (4.6), (4.7)

$$
\Delta V_{1 i}=-x_{i}^{2}-c y_{i}^{2}-2 \alpha x_{i} F_{i}-2 \beta y_{i} F_{i} .
$$

Put now

$$
q_{2}=\frac{1}{2} \sum_{l=1}^{2}\left|b_{l}\right| m_{l}\left(m_{l}+1\right), \quad S_{i}=\sum_{l=1}^{2}\left|b_{l}\right| \sum_{j=i-m_{l}}^{i-1}\left(j-i+1+m_{l}\right) y_{j}^{2} .
$$


Using (2.7) and $\lambda_{1}>0$ we have

$$
\begin{aligned}
2 x_{i} F_{i} & =2 \tau^{2} \sum_{l=1}^{2} b_{l} \sum_{j=i-m_{l}}^{i-1}\left(j-i+1+m_{l}\right) x_{i} y_{j} \\
& \leq \tau^{2} \sum_{l=1}^{2}\left|b_{l}\right| \sum_{j=i-m_{l}}^{i-1}\left(j-i+1+m_{l}\right)\left(\lambda_{1} x_{i}^{2}+\frac{1}{\lambda_{1}} y_{j}^{2}\right)=\lambda_{1} \tau^{2} q_{2} x_{i}^{2}+\frac{\tau^{2}}{\lambda_{1}} S_{i}
\end{aligned}
$$

and analogously

$$
2 y_{i} F_{i} \leq \lambda_{2} \tau^{2} q_{2} y_{i}^{2}+\frac{\tau^{2}}{\lambda_{2}} S_{i}, \quad \lambda_{2}>0
$$

As a result we obtain

$$
\Delta V_{1 i} \leq-\left(1-\alpha \tau^{2} \lambda_{1} q_{2}\right) x_{i}^{2}-\left(c-\beta \tau^{2} \lambda_{2} q_{2}\right) y_{i}^{2}+\rho S_{i}
$$

where

$$
\rho=\tau^{2}\left(\frac{\alpha}{\lambda_{1}}+\frac{\beta}{\lambda_{2}}\right)
$$

To neutralize the positive component in the estimate for $\Delta V_{1 i}$ choose $V_{2 i}$ in the form

$$
V_{2 i}=\frac{\rho}{2} \sum_{l=1}^{2}\left|b_{l}\right| \sum_{j=i-m_{l}}^{i-1}\left(j-i+\frac{3}{2}+m_{l}\right)^{2} y_{j}^{2}, \quad q_{3}=\frac{1}{2} \sum_{l=1}^{2}\left|b_{l}\right|\left(\frac{1}{2}+m_{l}\right)^{2} .
$$

Calculating $\Delta V_{2 i}$, we obtain

$$
\begin{aligned}
\Delta V_{2 i}=\frac{\rho}{2} \sum_{l=1}^{2}\left|b_{l}\right|\left[\sum_{j=i+1-m_{l}}^{i}\left(j-i+\frac{1}{2}+m_{l}\right)^{2} y_{j}^{2}-\sum_{j=i-m_{l}}^{i-1}\left(j-i+\frac{3}{2}+m_{l}\right)^{2} y_{j}^{2}\right] \\
=\frac{\rho}{2} \sum_{l=1}^{2}\left|b_{l}\right|\left[\left(\frac{1}{2}+m_{l}\right)^{2} y_{i}^{2}-\frac{1}{4} y_{i-m_{l}}^{2}\right. \\
\left.+\sum_{j=i-m_{l}}^{i-1}\left(\left(j-i+\frac{1}{2}+m_{l}\right)^{2}-\left(j-i+\frac{3}{2}+m_{l}\right)^{2}\right) y_{j}^{2}\right] \\
=\frac{\rho}{2} \sum_{l=1}^{2}\left|b_{l}\right|\left[\left(\frac{1}{2}+m_{l}\right)^{2} y_{i}^{2}-\frac{1}{4} y_{i-m_{l}}^{2}-2 \sum_{j=i-m_{l}}^{i-1}\left(j-i+1+m_{l}\right) y_{j}^{2}\right] \\
=\rho q_{3} y_{i}^{2}-\rho \sum_{l=1}^{2}\left|b_{l}\right|\left[\frac{1}{8} y_{i-m_{l}}^{2}+\sum_{j=i-m_{l}}^{i-1}\left(j-i+1+m_{l}\right) y_{j}^{2}\right] \leq \rho q_{3} y_{i}^{2}-\rho S_{i} .
\end{aligned}
$$

Thus, for the functional $V_{i}=V_{1 i}+V_{2 i}$ we have

$$
\Delta V_{i} \leq-\left(1-\alpha \tau^{2} \lambda_{1} q_{2}\right) x_{i}^{2}-\left(c-\beta \tau^{2} \lambda_{2} q_{2}-\rho q_{3}\right) y_{i}^{2}
$$


222 Stability of inverted pendulum

Using (4.14) we obtain the stability conditions in the form

$$
\tau^{2} \alpha \lambda_{1} q_{2}<1, \quad \tau^{2} \beta \lambda_{2} q_{2}+\tau^{2} q_{3}\left(\frac{\alpha}{\lambda_{1}}+\frac{\beta}{\lambda_{2}}\right)<c .
$$

To minimize the left-hand part of the second condition (4.18) put $\lambda_{2}=\sqrt{q_{3} / q_{2}}$. Then (4.18) takes the form

$$
\tau^{2} \alpha \lambda_{1} q_{2}<1, \quad \frac{\tau^{2}}{c}\left(2 \beta \sqrt{q_{2} q_{3}}+\frac{\alpha q_{3}}{\lambda_{1}}\right)<1 .
$$

Choosing $\lambda_{1}>0$ from the condition

$$
\alpha \lambda_{1} q_{2}=\frac{1}{c}\left(2 \beta \sqrt{q_{2} q_{3}}+\frac{\alpha q_{3}}{\lambda_{1}}\right)
$$

we obtain

$$
\lambda_{1}=\frac{\sqrt{q_{3}}\left(\beta+\sqrt{\beta^{2}+\alpha^{2} c}\right)}{\alpha c \sqrt{q_{2}}} .
$$

Substituting (4.21) into (4.19) we get stability condition in the form

$$
\gamma\left(\beta+\sqrt{\beta^{2}+\alpha^{2} c}\right)<c, \quad \gamma=\tau^{2} \sqrt{q_{2} q_{3}} .
$$

From here it follows

$$
\gamma\left(\gamma \alpha^{2}+2 \beta\right)<c
$$

Put

$$
\hat{k}_{i}=\sum_{l=1}^{2}\left|b_{l}\right| h_{l}^{i}, \quad i=0,1,2
$$

Then

$$
\begin{aligned}
& q_{2} \tau^{2}=\frac{1}{2} \sum_{l=1}^{2}\left|b_{l}\right| h_{l}\left(\tau+h_{l}\right)=\frac{1}{2}\left(\tau \hat{k}_{1}+\hat{k}_{2}\right), \\
& q_{3} \tau^{2}=\frac{1}{2} \sum_{l=1}^{2}\left|b_{l}\right|\left(\frac{\tau}{2}+h_{l}\right)^{2}=\frac{1}{2}\left(\frac{\tau^{2}}{4} \hat{k}_{0}+\tau \hat{k}_{1}+\hat{k}_{2}\right) .
\end{aligned}
$$


Therefore,

$$
\gamma=\frac{1}{2} \sqrt{\left(\tau \hat{k}_{1}+\hat{k}_{2}\right)\left(\frac{\tau^{2}}{4} \hat{k}_{0}+\tau \hat{k}_{1}+\hat{k}_{2}\right)} .
$$

Using dependence $\alpha$ and $\beta$ on $c$ put

$$
\alpha=B^{-1}\left(A+2 a_{1} c\right), \quad \beta=B^{-1}(\tau+G c),
$$

where

$$
A=2-\tau\left(k_{1}-a_{1} \tau\right), \quad B=\left(k_{1}-a_{1} \tau\right)(4-G \tau), \quad G=2 k_{1}-a_{1} \tau .
$$

Substituting (4.27) into (4.23) we obtain

$$
\gamma B^{-2}\left(\frac{A^{2} \gamma+2 B \tau}{c}+4 \gamma a_{1}^{2} c+4 A \gamma a_{1}+2 B G\right)<1 .
$$

After minimization of the left-hand part of (4.29) with respect to $c>0$ one can rewrite (4.29) in the form

$$
\delta(\tau)=2 \gamma B^{-2}\left(2 a_{1} \sqrt{\gamma\left(A^{2} \gamma+2 B \tau\right)}+2 A \gamma a_{1}+B G\right)<1 .
$$

One has remember that in condition (4.30) $a_{1}$ is defined by (1.2), $A, B, G$ are defined by (4.28) and $\gamma$ is defined by (4.26), (4.24). So, $A, B, G$ and $\gamma$ depend on $\tau$.

Thus, the following theorem is proven.

Theorem 4.1. Let conditions (1.5) hold and the step of quantization $\tau>0$ satisfies condition (4.30). Then the trivial solution of system (2.3) is asymptotically stable.

LEMMA 4.2. If condition (1.6) holds then there exists enough small $\tau>0$ that condition (4.30) holds too.

Proof. For $\tau=0$ condition (4.30) takes the form

$$
\hat{k}_{2}<4\left(1+\sqrt{1+\frac{4 a_{1}}{k_{1}^{2}}}\right)^{-1} .
$$

It is easy to see that if condition (1.6) holds then condition (4.31) (or condition (4.30) for $\tau=0$ ) holds too. Since the function $\delta(\tau)$ is continuous in the point $\tau=0$ then if condition (4.30) holds for $\tau=0$ then it holds for enough small $\tau>0$ also. The proof is completed.

Corollary 4.3. Let conditions (1.5), (1.6) hold then there exists enough small $\tau>0$ that the trivial solution of system (2.3) is asymptotically stable.

\section{Numerical analysis}

Here we consider some numerical examples which illustrate the theoretical results obtained above. For illustration of Corollary 4.3 consider the following example. 
224 Stability of inverted pendulum

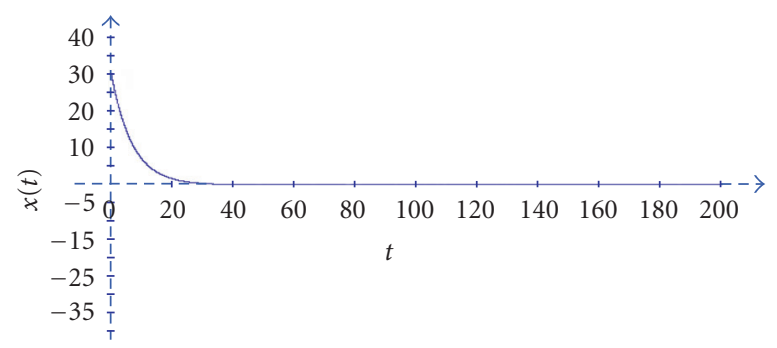

Figure 5.1

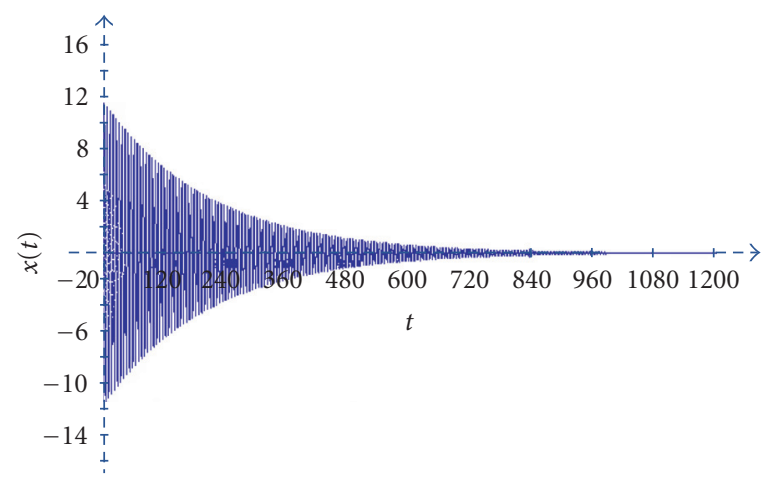

Figure 5.2

Example 5.1. Put in equation (1.7) $a=9.5, b_{1}=10, b_{2}=-20, h_{1}=0.4, h_{2}=0.02$. Then $a_{1}=0.5>0, k_{1}=3.6>0, \hat{k}_{2}=1.608<k_{m}=1.92$. Conditions (1.5), (1.6) hold, therefore (Theorem 1.2), the trivial solution of equation (1.7) is asymptotically stable. Besides there exists enough small $\tau>0$ that condition (4.30) holds. Using $\tau=0.01$ we obtain $\delta(0.01)=0.869<1$, that is condition (4.30) holds. Therefore, the trivial solution of difference system (2.3) is asymptotically stable. On Figure 5.1 it is shown that the trajectory of solution of system (2.3) with the initial condition $x_{j}=33, j \leq 0, y_{0}=0$ goes to zero.

If conditions (1.5) hold but condition (1.6) does not hold then the trivial solution of equation (1.7) can be asymptotically stable or unstable. If in this case for some $\tau>0$ condition (4.30) does not hold too then the trivial solution of difference system (2.3) can be also asymptotically stable or unstable. In the following two examples one can see the both situations.

Example 5.2. Put in equation (1.7) $a=3, b_{1}=1, b_{2}=-5, h_{1}=0.55, h_{2}=0.1$. Then $a_{1}=$ $1>0, k_{1}=0.05>0, \hat{k}_{2}=0.3525>k_{m}=0.0975, \delta(0.01)=20.83>1$. So, conditions $(1.5)$ hold but conditions (1.6) and (4.30) do not hold. On Figure 5.2 it is shown that the trajectory of solution of system (2.3) with the initial condition $x_{j}=12, j \leq 0, y_{0}=0$ goes to zero. 


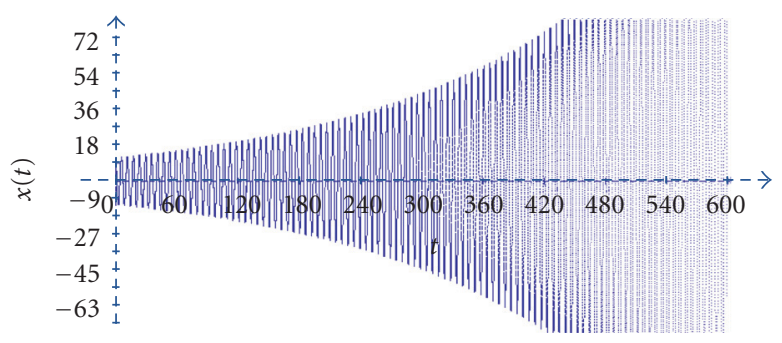

Figure 5.3

Example 5.3. Putting in the previous example $h_{1}=0.53$ (without changing the values of the other parameters) we obtain $a_{1}=1>0, k_{1}=0.03>0, \hat{k}_{2}=0.3309>k_{m}=0.0591$, $\delta(0.01)=73.06>1$. As in the previous example conditions (1.5) hold, conditions (1.6) and (4.30) do not hold but in this case the trajectory of solution of system (2.3) with the initial condition $x_{j}=12, j \leq 0, y_{0}=0$ goes to infinity (Figure 5.3).

\section{References}

[1] D. J. Acheson, A pendulum theorem, Proc. Roy. Soc. London Ser. A 443 (1993), no. 1917, 239245.

[2] D. J. Acheson and T. Mullin, Upside-down pendulums, Nature 366 (1993), no. 6452, 215-216.

[3] J. A. Blackburn, H. J. T. Smith, and N. Grønbech-Jensen, Stability and Hopf bifurcations in an inverted pendulum, Amer. J. Phys. 60 (1992), no. 10, 903-908.

[4] P. Borne, V. B. Kolmanovskii, and L. E. Shaikhet, Stabilization of inverted pendulum by control with delay, Dynam. Systems Appl. 9 (2000), no. 4, 501-514.

[5] P. L. Kapitza, Dynamical stability of a pendulum when its point of suspension vibrates, and Pendulum with a vibrating suspension, In Collected Papers of P. L. Kapitza (D. ter Haar, ed.), vol. 2, Pergamon Press, London, 1965, pp. 714-737.

[6] V. B. Kolmanovskii and L. E. Shaikhet, New results in stability theory for stochastic functionaldifferential equations (SFDEs) and their applications, Proceedings of Dynamic Systems and Applications, Vol. 1 (Atlanta, GA, 1993), Dynamic, Georgia, 1994, pp. 167-171.

[7] - General method of Lyapunov functionals construction for stability investigation of stochastic difference equations, Dynamical Systems and Applications, World Sci. Ser. Appl. Anal., vol. 4, World Scientific Publishing, New Jersey, 1995, pp. 397-439.

[8] Method for constructing Lyapunov functionals for stochastic differential equations of neutral type, Differential Equations 31 (1995), no. 11, 1819-1825.

[9] _ Construction of Lyapunov functionals for stochastic hereditary systems: a survey of some recent results, Math. Comput. Modelling 36 (2002), no. 6, 691-716.

[10] Some peculiarities of the general method of Lyapunov functionals construction, Appl. Math. Lett. 15 (2002), no. 3, 355-360.

[11] About one application of the general method of Lyapunov functionals construction, Internat. J. Robust Nonlinear Control 13 (2003), no. 9, 805-818, Special Issue on Time Delay Systems.

[12] About some features of general method of Lyapunov functionals construction, Stab. Control Theory Appl. 6 (2004), no. 1, 49-76.

[13] M. Levi, Stability of the inverted pendulum - a topological explanation, SIAM Rev. 30 (1988), no. 4, 639-644. 


\section{Stability of inverted pendulum}

[14] M. Levi and W. Weckesser, Stabilization of the inverted linearized pendulum by high frequency vibrations, SIAM Rev. 37 (1995), no. 2, 219-223.

[15] L. E. Shaikhet, Necessary and sufficient conditions of asymptotic mean square stability for stochastic linear difference equations, Appl. Math. Lett. 10 (1997), no. 3, 111-115.

L. Shaikhet: Department of Higher Mathematics, Donetsk State University of Management, Chelyuskintsev 163-a, Donetsk 83015, Ukraine

E-mail addresses: leonid@dsum.edu.ua; leonid.shaikhet@usa.net 


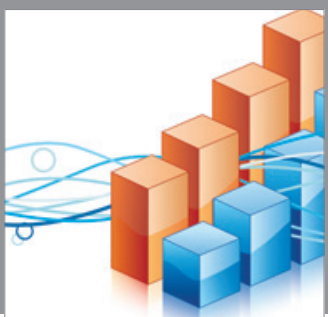

Advances in

Operations Research

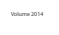

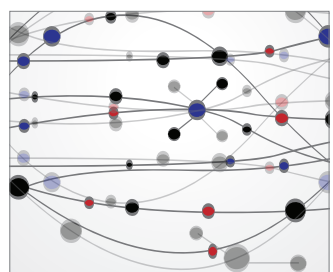

\section{The Scientific} World Journal
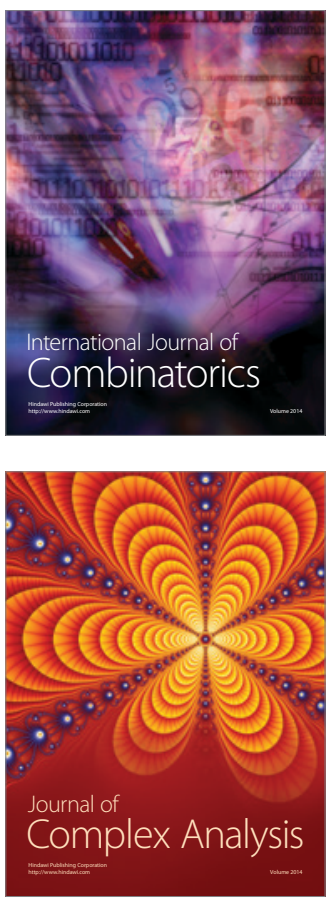

International Journal of

Mathematics and

Mathematical

Sciences
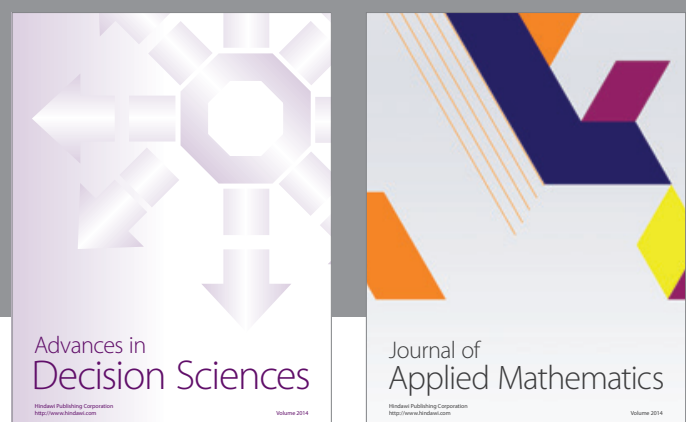

Journal of

Applied Mathematics
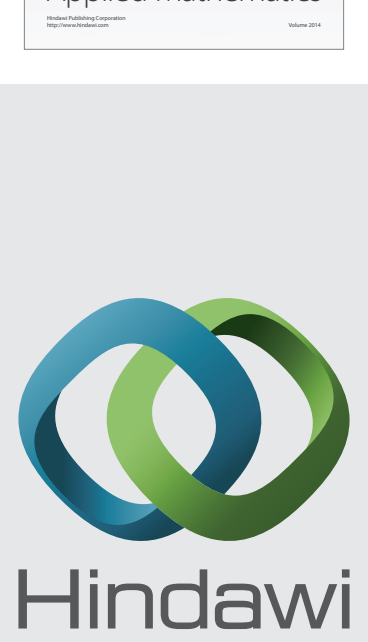

Submit your manuscripts at http://www.hindawi.com
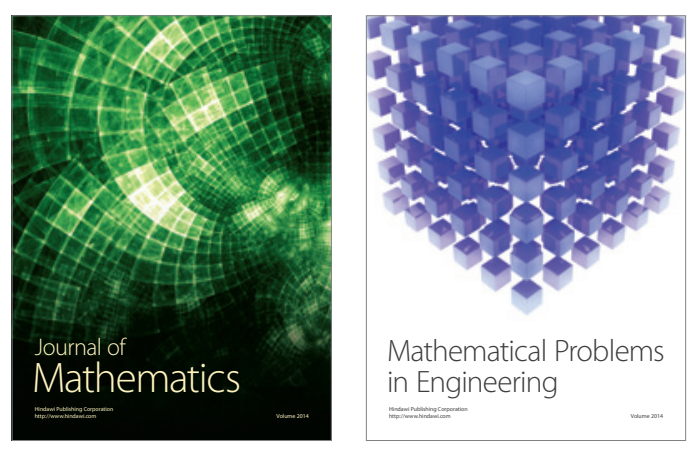

Mathematical Problems in Engineering
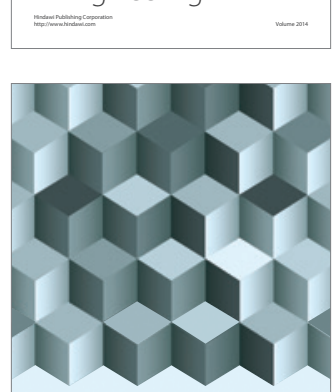

Journal of

Function Spaces
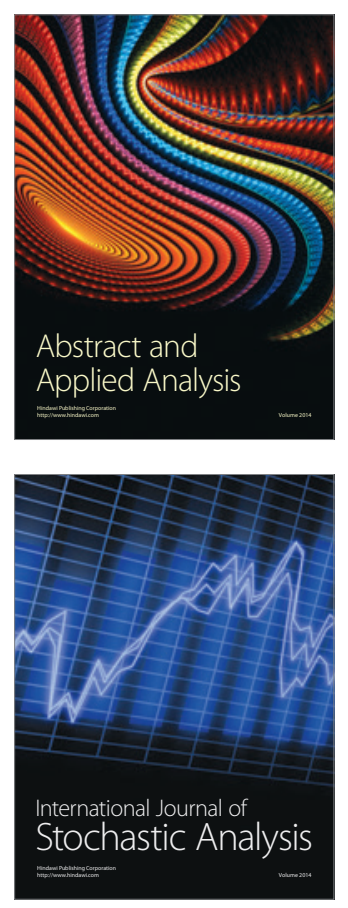

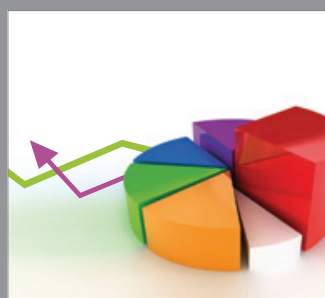

ournal of

Probability and Statistics

Promensencen
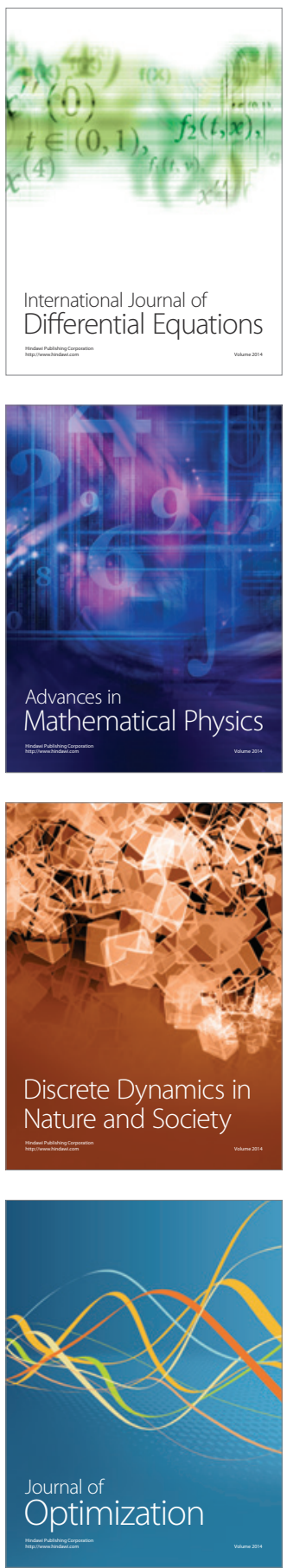